\title{
Genes encoding cytoplasmic intermediate filament proteins of vertebrates revisited: Identification of a cytoplasmic intermediate filament protein in the sea anemone Nematostella
}

\author{
Alexander Zimek ${ }^{\mathrm{a}}$, Sören Thiering ${ }^{\mathrm{b}}$, Klaus Weber ${ }^{\mathrm{a}}$, Thomas M. Magin ${ }^{\mathrm{b}, *}$ \\ a Max Planck Institute for Biophysical Chemistry, 37077 Goettingen, Germany \\ ${ }^{\mathrm{b}}$ Division of Cell and Developmental Biology, Translational Centre for Regenerative Medicine (TRM) and Biology, University of Leipzig, Leipzig, Germany
}

\section{A R T I C L E I N F O}

\section{Article history:}

Received 25 March 2012

Received in revised form 31 July 2012

Accepted 3 August 2012

\section{Keywords:}

Metazoa

Molecular phylogeny

Hemichordates

Cnidaria

Radiata

\begin{abstract}
A B S T R A C T
The cytoskeleton is crucial in determining cell architecture, division, motility, transport processes and in local control of signal transduction. Relatives of actin and tubulin are expressed in all phyla, underlining the fundamental importance of conserved cytoskeletal functions. Intermediate filament proteins have evolved in parallel with tissue diversity in the animal kingdom, likely from the demand to adapt one class of cytoskeletal proteins to cell type-restricted functions. Up to now, the evolutionary origin of cytoplasmic intermediate filament proteins remains unknown. Using a known gene encoding a cytoplasmic intermediate filament protein from the hemichordate Saccoglossus kowalevskii, we have identified the first corresponding gene in the sea anemone Nematostella, tentatively named cytovec. Our data reveal a relationship of cytovec with Hydra vulgaris nematocilins A and B that also lack a CAAX box. In light of additional recent findings, our data show that cytoplasmic intermediate filament genes are present in the common ancestor of Cnidaria and Bilateria.
\end{abstract}

(C) 2012 Elsevier GmbH. All rights reserved.

\section{Introduction}

The cytoskeleton is crucial in determining cell architecture, division, motility transport processes and in local control of signal transduction. Whereas relatives of actin and tubulin are expressed in all phyla intermediate filament proteins have evolved in parallel with tissue diversity in the animal kingdom, likely from the demand to adapt one class of cytoskeletal proteins to cell typerestricted functions (Fuchs and Weber, 1994; Wickstead and Gull, 2011). The multigene family of metazoan intermediate filament proteins covers two groups: the nuclear lamins and the cytoplasmic intermediate filament (IF) proteins. Their sequence homologies are particularly obvious in the protostomic IF proteins, because the central coiled coil domains have the same length as the lamins, while in deuterostomic species the central domain of IF proteins is shortened (Dodemont et al., 1990; Doring and Stick, 1990).

A major difference between lamins and IF proteins concerns two features of the lamins: a nuclear localization signal, usually 4 consecutive basic residues in the carboxy-terminal tail domain, and the

\footnotetext{
* Corresponding author at: Translational Centre for Regenerative Medicine (TRM) and Institute of Biology, Cell and Developmental Biology, University of Leipzig, Talstrasse 33, D-04103 Leipzig, Germany.

Tel.: +49034197 39662; fax: +4903419739609.

E-mail address: thomas.magin@trm.uni-leipzig.de (T.M. Magin).
}

C-terminal isoprenylation site $\mathrm{C}$-a-a $\mathrm{M}$, which in a stepwise reaction gives rise to chargeless carboxy terminal cysteine (Gruenbaum et al., 2005).

RNA interference studies under standard conditions showed that at least 5 of the IF proteins of the nematode C. elegans are essential in the earliest stages of embryonic development (Karabinos et al., 2001). The family of mammalian cytoplasmic IF proteins is much larger (Hesse et al., 2001) and its expression patterns has been reviewed (Iwatsuki and Suda, 2010; Windoffer et al., 2011). In accordance with the large number of mammalian IF genes, at least 25 human IF genes show mutations which give rise or contribute to diseases (Bonifas et al., 1991; Vassar et al., 1991; Szeverenyi et al., 2008; http://www.interfil.org/index.php). While type III proteins like desmin form in vivo and in vitro homopolymeric IF, the epithelial keratins are obligatory heteropolymers based on dimers containing a type I and a type II keratin. The type IV neurofilament proteins can have larger molecular weights due to unique extensions of the C-terminal tail domains.

Metazoa cover the more basal phyla of the radiata, which have radial symmetry and the large group of bilateral animals, which have bilateral symmetry (Wehner and Gehring, 2007). When genomic information became available for the first representative of the radiata, we screened the developing genome of the sea anemone Nematostella vectensis for representatives of lamin and IF genes. Although we found a lamin gene, we and others failed to detect IF genes (Zimek and Weber, 2008). This was unexpected, 


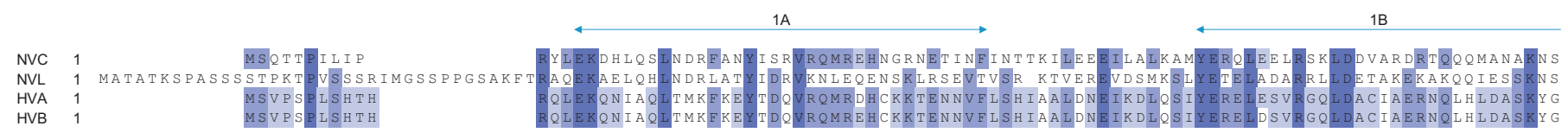

$1 \mathrm{~B}$

$2 \mathrm{~A}$

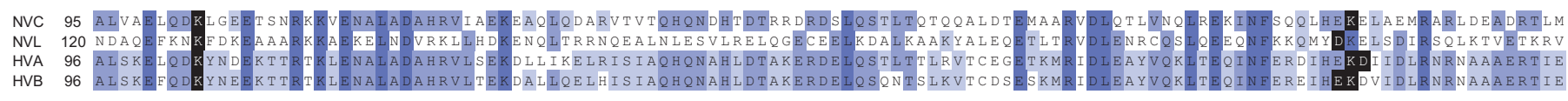

$2 \mathrm{~A}$

$2 B$

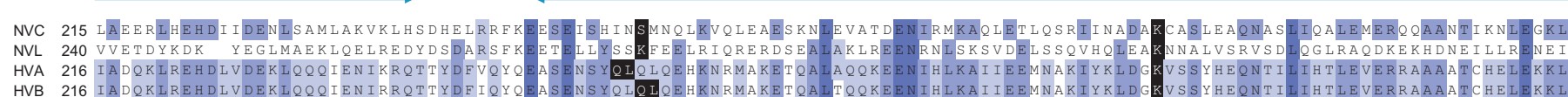
$2 \mathrm{~B}$

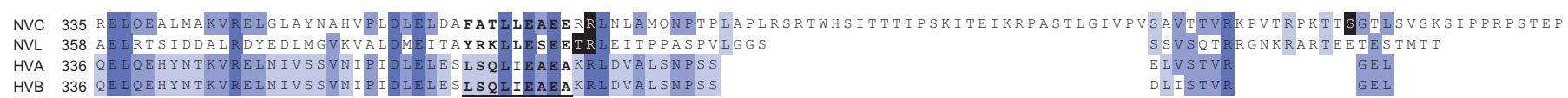

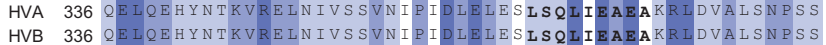

Ig-fold

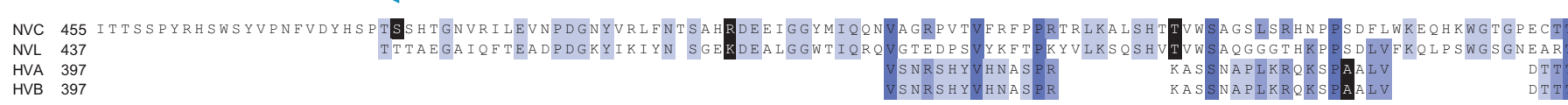
$\longrightarrow$

NVC 575 ILCKPNGQAMATTAAFPEGVPNQIPTRDTGPTSPRDGEQLNENTGPMSPR

NVL 533 ALVNAGGEEMATLEE

$\begin{array}{lll}\text { HVA } & 433 & \text { E L P P L } \\ \text { HVB } & 433 & \text { ELPPL }\end{array}$

RYKTPEPIPREIEGKPESRPPTDPLHPHSVQSRVKAGGHDGMTVNPQSRSQTTRPDPAGATRTGGAPI G AP L

CaaX-box

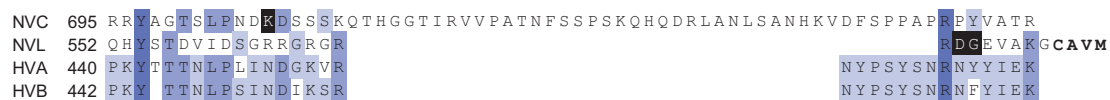

NVC Nematostellavectensiscytovec

NVL Nematostellavectensis lamin

HVA Hydra vulgaris nematocilin A (BAG48261.1)

HVB Hydra vulgaris nematocilin B (BAG48262.1)

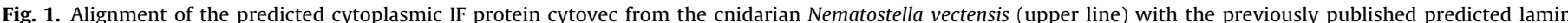

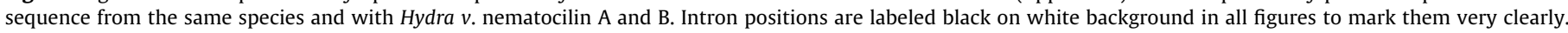

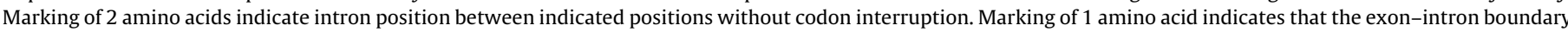

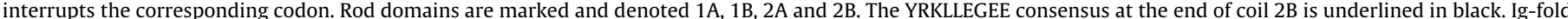

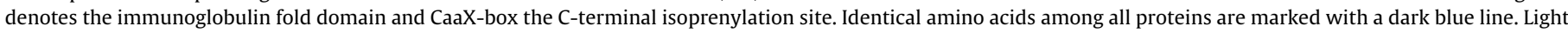

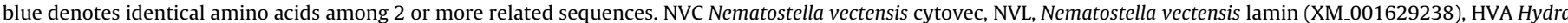

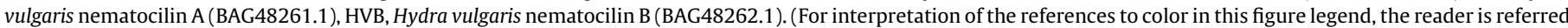
to the web version of the article.)

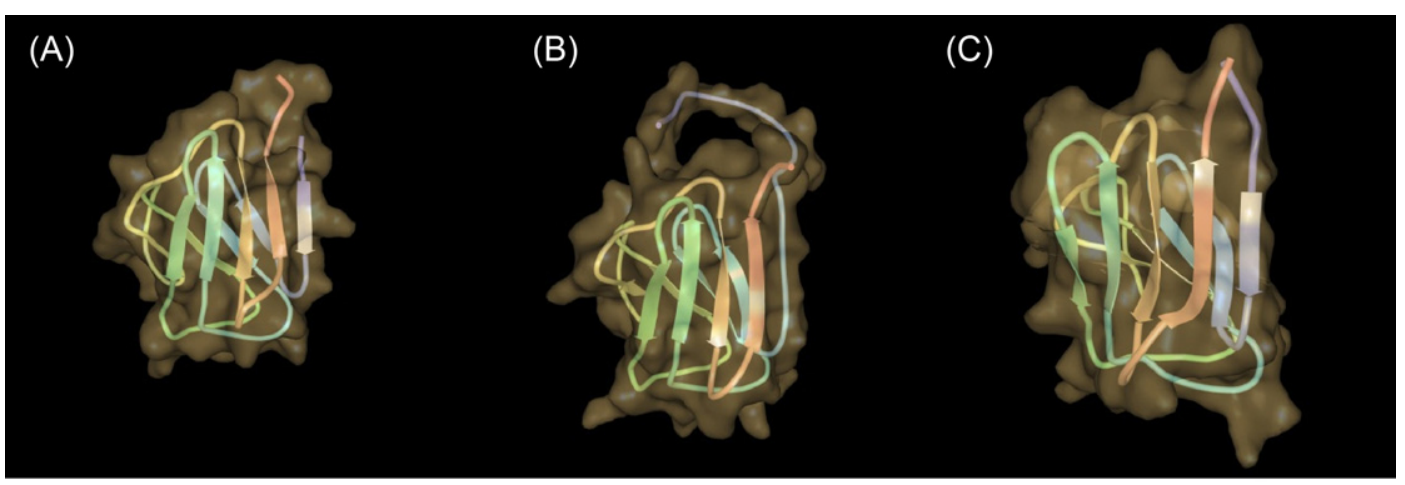

(A) Homo sapiens lamin $\mathrm{A} / \mathrm{C}$

(B) Nematostellavectensiscytovec

(C) Nematostellavectensislamin

Fig. 2. Structure of Homo sapiens lamin A/C Ig fold and predicted structure of corresponding domain in Nematostella vectensis cytovec and lamin. 
Table 1

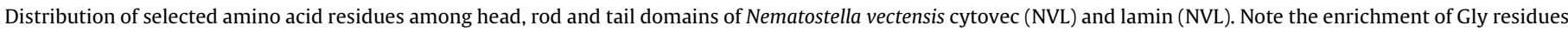

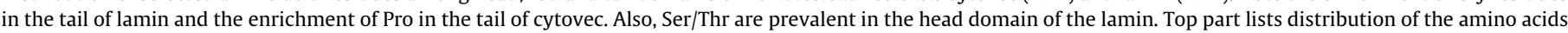

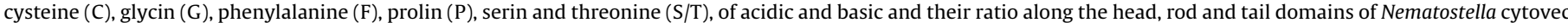
(NVC) and lamin. Lower part, graphical display of amino acid distribution along head, rod, tail and entire protein.

\begin{tabular}{|c|c|c|c|c|c|c|c|c|}
\hline & \multicolumn{4}{|l|}{ NVC } & \multicolumn{4}{|l|}{ NVL } \\
\hline & Head (\%) & $\operatorname{Rod}(\%)$ & Tail (\%) & Whole (\%) & Head (\%) & $\operatorname{Rod}(\%)$ & Tail (\%) & Whole (\%) \\
\hline C & 0.0 & 0.3 & 1.1 & 0.5 & 0.0 & 0.5 & 0.6 & 0.5 \\
\hline G & 0.0 & 1.1 & 5.6 & 2.4 & 5.6 & 1.1 & 11.8 & 4.7 \\
\hline $\mathrm{F}$ & 2.8 & 1.1 & 2.8 & 1.7 & 2.8 & 1.4 & 2.2 & 1.7 \\
\hline $\mathrm{P}$ & 5.6 & 1.4 & 10.7 & 4.5 & 13.9 & 0.0 & 5.1 & 2.4 \\
\hline $\mathrm{S} / \mathrm{T}$ & 13.9 & 11.5 & 23.6 & 15.3 & 44.4 & 11.2 & 19.1 & 15.7 \\
\hline Acidic & 8.3 & 17.8 & 6.2 & 13.6 & 0.0 & 23.2 & 12.4 & 18.4 \\
\hline Basic & 19.4 & 16.4 & 16.3 & 16.6 & 11.1 & 19.7 & 14.0 & 17.4 \\
\hline Acidic/basic & 27.8 & 34.2 & 22.5 & 30.2 & 11.1 & 42.9 & 26.4 & 35.9 \\
\hline
\end{tabular}

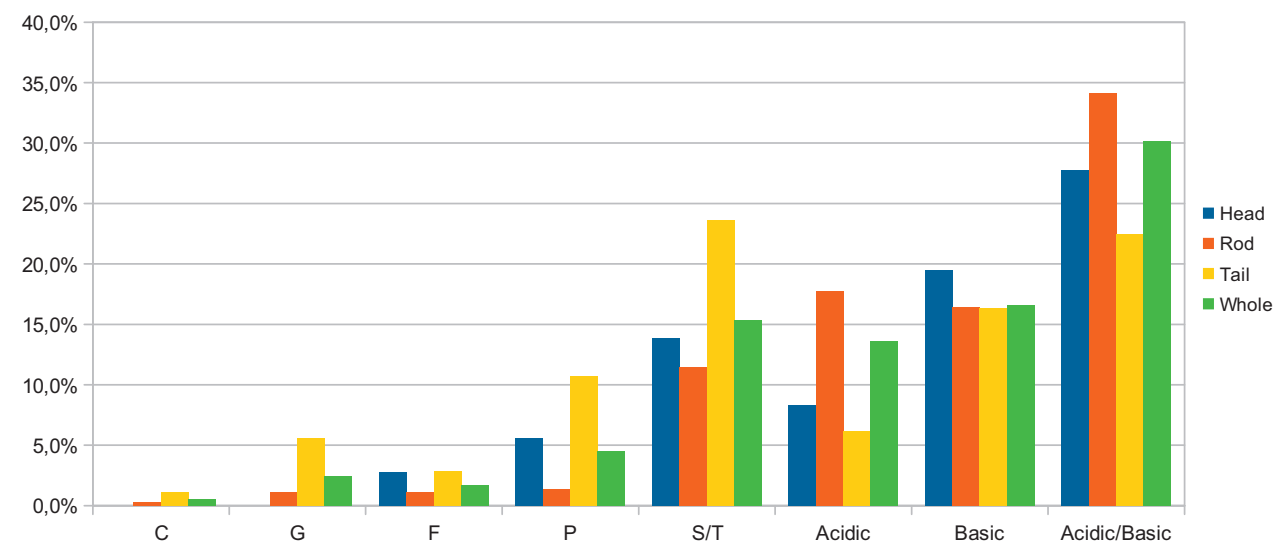

NVL

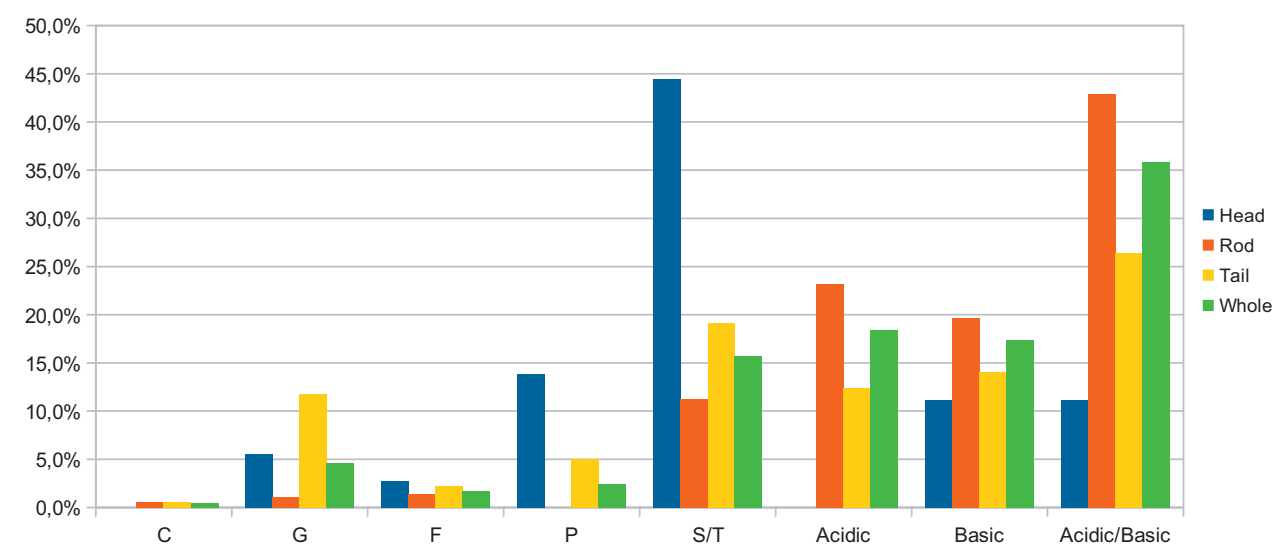

since previous electron microscopical studies documented a wealth of cytoplasmic IF in the radiata (see for instance Bartnik and Weber (1989)). Here we repeated the screen using however as probe a gene from metazoa closer related to radiata than the mammalian genes, which were earlier used. In our work, we identified the cytoplasmic protein cytovec, a putative IF-forming protein with the highest degree of similarity to the cytosolic IF protein from Clytia hemispherica and from nematocilin A and B from Hydra vulgaris (Hwang et al., 2008).

\section{Materials and methods}

We analyzed the genome assembly of the sea anemone ( $N$. vectensis). The Nematostella genome assembly 1.0 was produced by the Joint Genome Institute (JGI). It has a 7.8-fold coverage in 10,804 scaffolds with a total sequence length of $356 \mathrm{Mbp}$ of sequence. Roughly half of the genome is contained in 181 scaffolds all at least $473 \mathrm{~kb}$ in length. Graphics were created with the open source suite LibreOffice, available at http://www.documentfoundation.org. To compare the $N$. vectensis cytovec with other metazoans IF, we used the following protein sequences: Acropora digitifera (adi_v1.13506), C. hemispherica (CL437Contig1), Caenorhabditis elegans: Intermediate filament protein ifa-1 (P991), ifb-1 (Q19289), ifc-1 (045168), ifd-1 (Q86DC6), lamin (Q21443), Ciona intestinalis cIF (XP_002120583.1), nuclear lamin (NP_001093902.1), Danio rerio lamin A (AAI63799.1), Drosophila melanogaster lamin C (NP_523742.2), Homo sapiens desmin (NP_001918.3), keratin type I cytoskeletal 18 (NP_954657.1), keratin type II cytoskeletal 8 isoform 


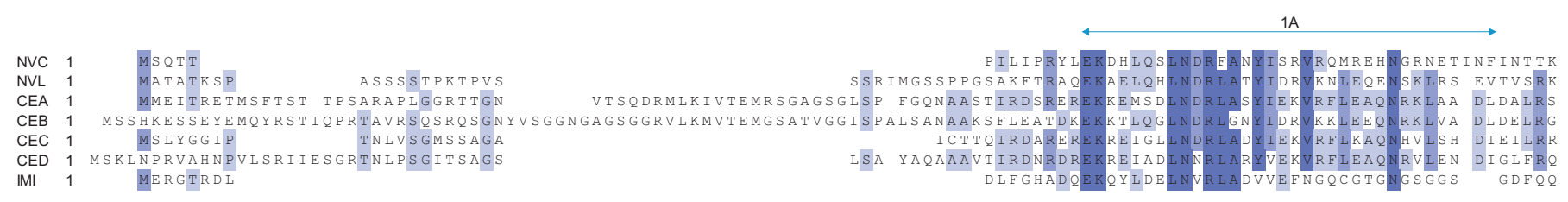

$1 \mathrm{~B}$

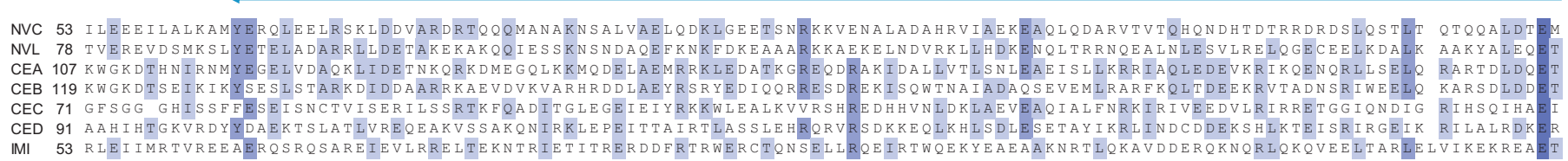
$\stackrel{1 \mathrm{~B}}{\longrightarrow}$ $2 \mathrm{~A}$

$2 \mathrm{~B}$

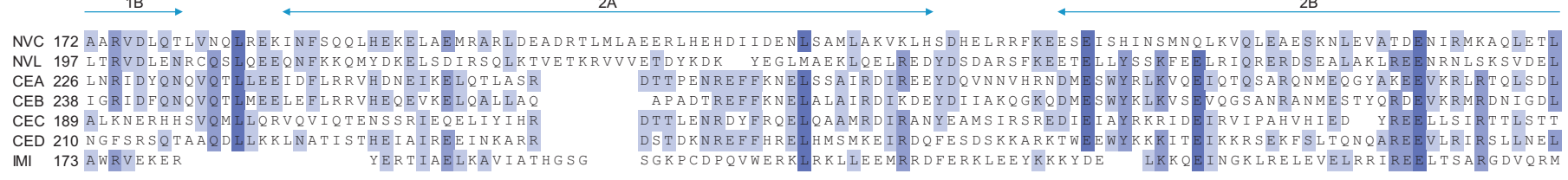
2B

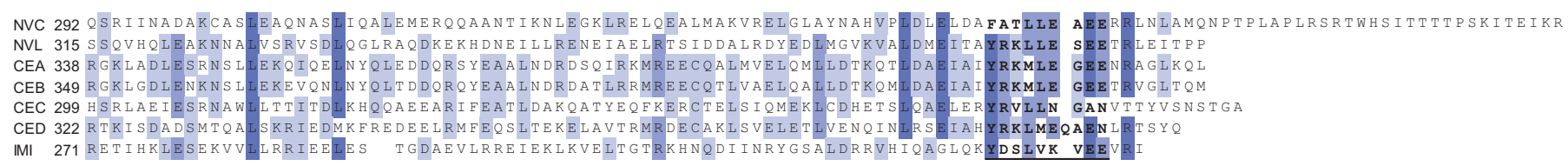

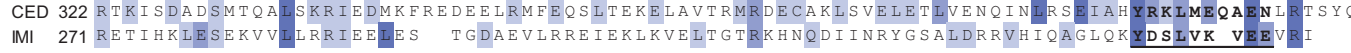

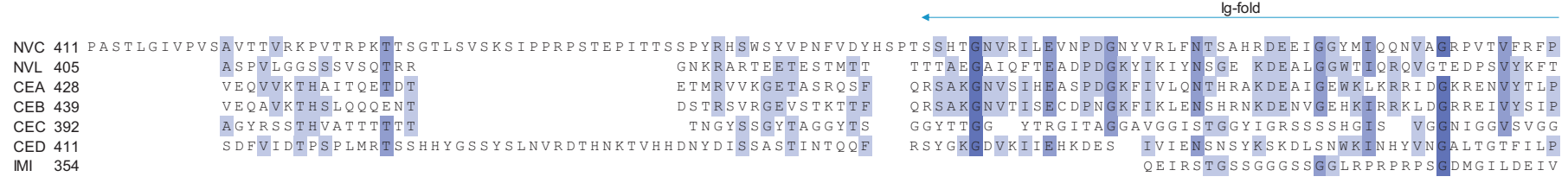
lg-fold

NVC 531 PRTRLKALSHTVWSAGSISRHNPPDELWKQHKWGTGPCTTILCKPNGQAMAWTTAAFPFGVRQIPTRDTGPTSPRDSGEQLNENTGPMSPRYKTPEPIPREIEGKPESRPPTDP NVL 489 PKYVLKSQSHVTVWSAQGGTHRPSDLVEKQLSWGSGNEATALVNAGGEMATLLEKVEQHYSTDIDSGRRRGRDGEVAKGCAVM

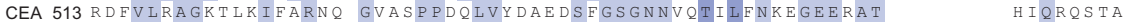

CEB 524 ANVIIRGRITIYARQGinNPESLVEDENTWGIGANVVTSLVNKDGERAT HTRIQSGQ

CEC 472 HINGYHASGDISAAGRHH ESYSYSSSNN

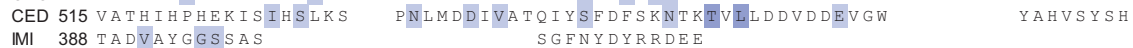

NVC 651 LHPHSVOSRVKAGGHDGMTVNPOSRSOTTRPDPAGATRTGGAPLRRYAGTSLPNDKDSSSKOTHGGTIRVVPATNFSSPSKOHODRLANLSANHKVDFSPPAPRPYVATR

NVL

CEA

CEB

CED

IMI

NVC Nematostellavectensiscytovec

NVL Nematostellavectensis lamin

CEA Caenorhabditis elegans Intermediate filament protein ifa-1 (P991)

CEB Caenorhabditis elegans Intermediate filament protein ifb-1 (Q19289)

CEC Caenorhabditis elegans Intermediate filament protein ifc-1 (O45168)

CED Caenorhabditis elegans Intermediate filament protein ifd-1 (Q86DC6)

IMI Isotomurusmaculatusisomin (ACJ66847.1)

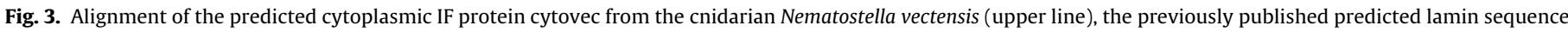

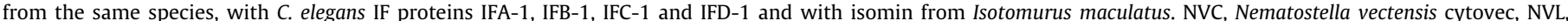

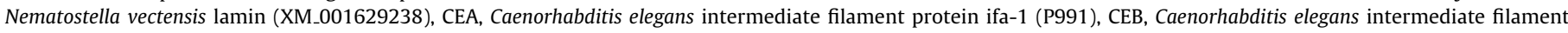

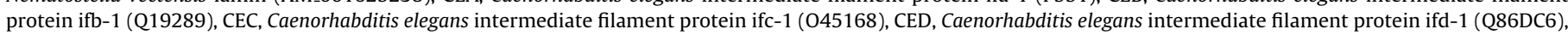
IMI, Isotomurus maculatus isomin (ACJ66847.1).

2 (NP_002264.1), lamin A/C transcript variant 1 (AAW32540.1), neurofilament light polypeptide (NP_006149.2), vimentin (NP_003371.2), H. vulgaris nematocilin A (BAG48261.1) nematocilin B (BAG48262.1), Isotomurus maculatus isomin (ACJ66847.1), N. vectensis lamin (XM_001629238), Saccoglossus kowalevskii cIF (XP_002736176.1), lamin A/C-like (XP_002734960.1), Strongylocentrotus purpuratus cIF (XP_796075.2), nuclear intermediate filament protein (NP_999665.1), Xenopus (Silurana) tropicalis lamin A/C (NP_001039148.1). These sequences were either man- ually selected or determined by the NCBI BLASTP search tool (http://blast.ncbi.nlm.nih.gov/Blast.cgi). For the BLASTP search we used the $N$. vectensis cytovec sequence.

The sequence alignments were calculated by using the multiple alignment tool MUSCLE v3.8.31 (http://www.drive5.com/muscle/). For further visualizing of these alignments we used the Jalview $\mathrm{v}$ 2.7 tool (http://www.jalview.org/).

Protein structures were predicted by the phyre 2 tool (http://www.sbg.bio.ic.ac.uk/ phyre2/html/page.cgi?id=index) 


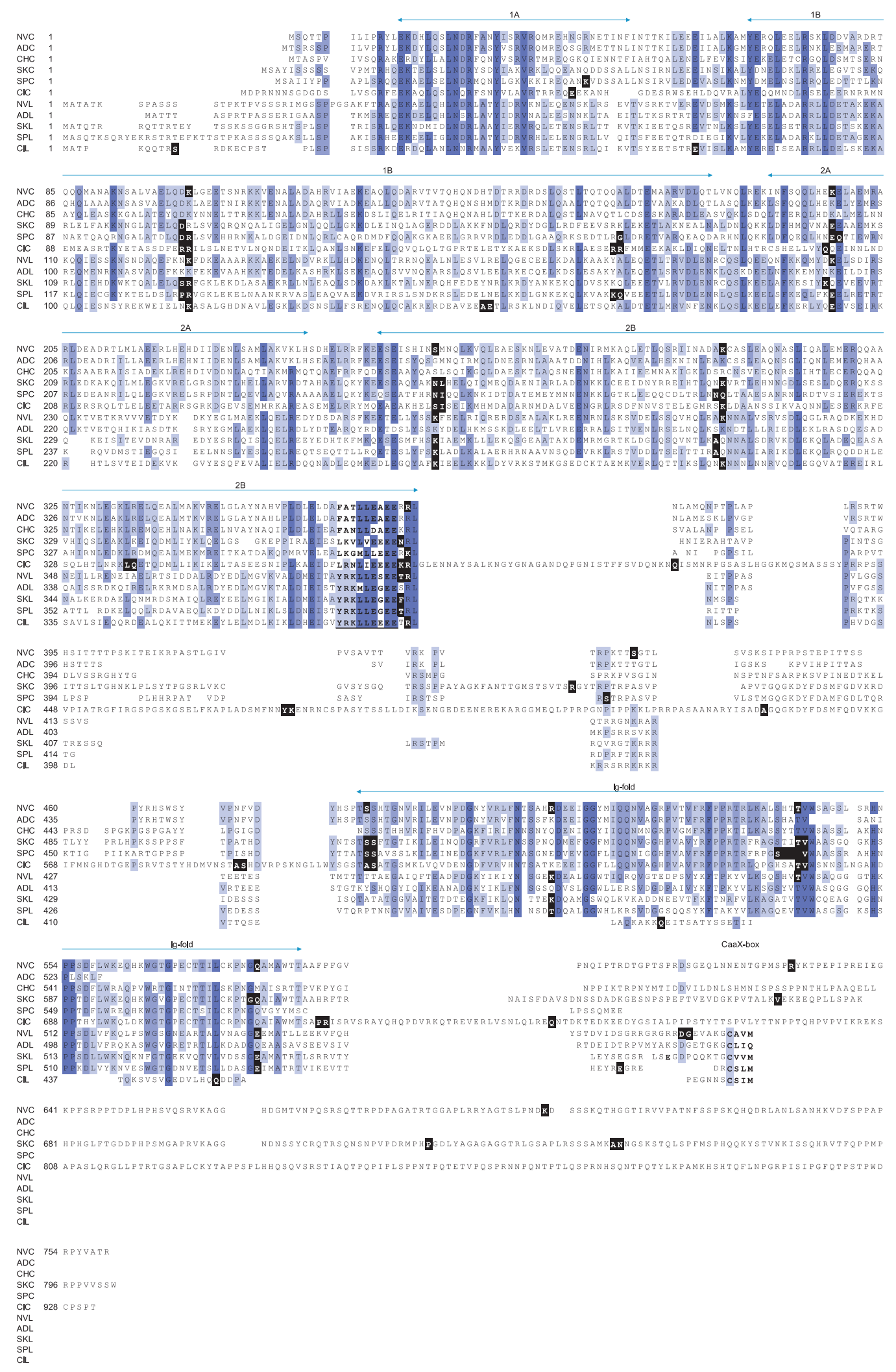

Fig. 4. Alignment of the predicted cytoplasmic IF protein protein cytovec from the cnidarian Nematostella vectensis (upper line) with predicted cytoplasmic IF proteins from ADC, Acropora digitifera* (adi_v1.13506), CHC, Clytia hemispherica. (CL437Contig1), SKC, Saccoglossus kowalevskii (XP_002736176.1), SPC, Strongylocentrotus purpuratus (XP_796075.2), CIC, Ciona intestinalis (XP_002120583.1), NVL, Nematostella vectensis lamin, ADL, Acropora digitifera lamin*(adi_v1.19659), SKL, Saccoglossus kowalevskii laminA/C-like (XP_002734960.1), SPL, Strongylocentrotus purpuratus nuclear intermediate filament protein (NP_999665.1), CIL, Ciona intestinalis nuclear lamin (NP_001093902.1). *For Clytia hemispherica and Acropora digitifera, only incomplete transcriptome data are available, precluding to mark exon overlaps. 


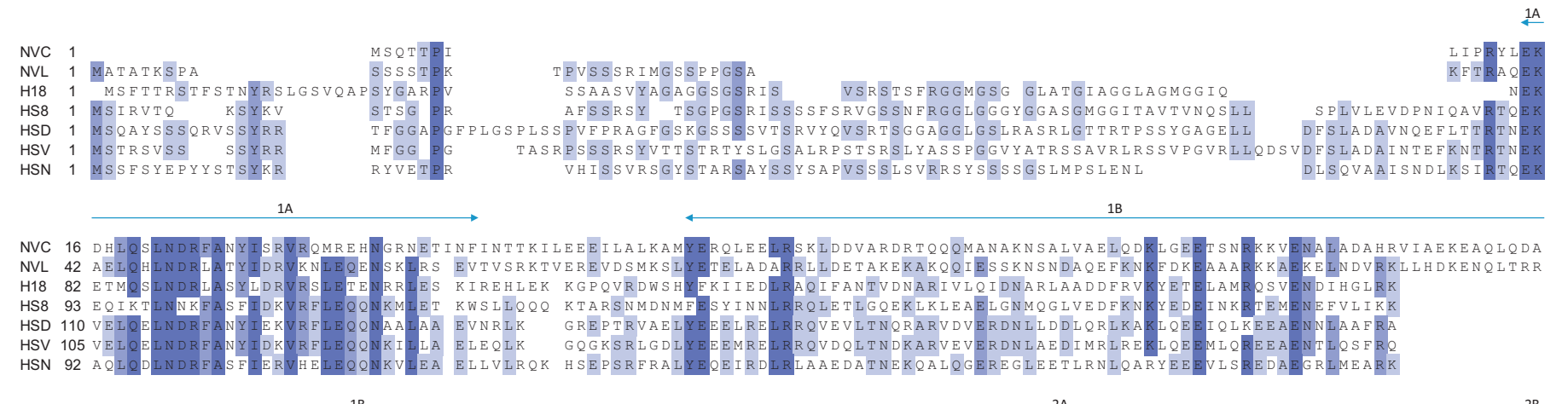

$1 \mathrm{~B}$

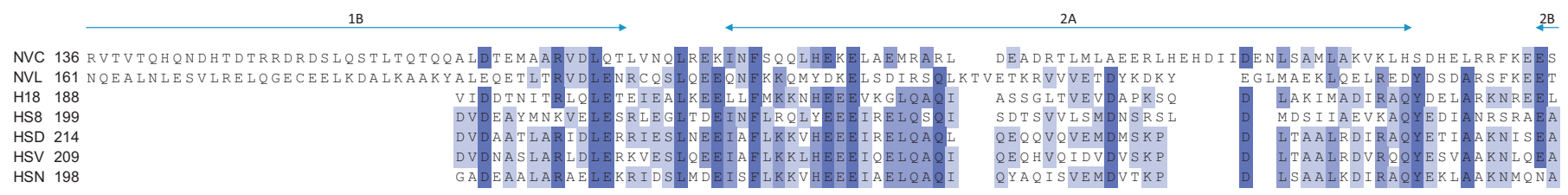

$2 B$

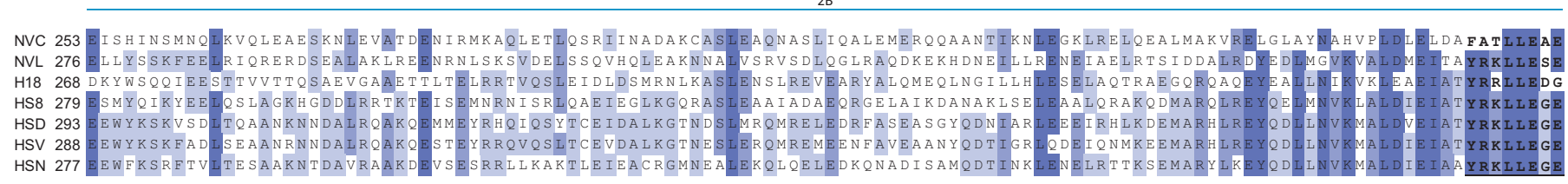

$\stackrel{2 \mathrm{~B}}{\longrightarrow}$

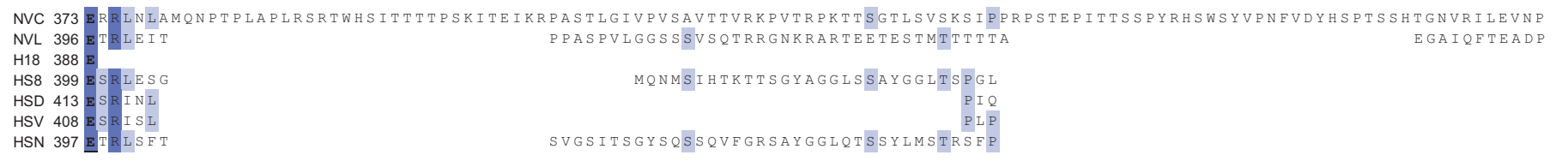

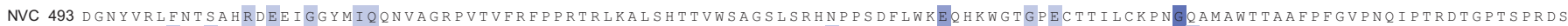
NVL 452 DGKYIKIYNSGE KDEALGGWTIOROVGTEDPSVYKFTPKYVLKSOSHVTVWSA OGGGTHKPPSDLVFKOLPSWGSGEARTALVNAG

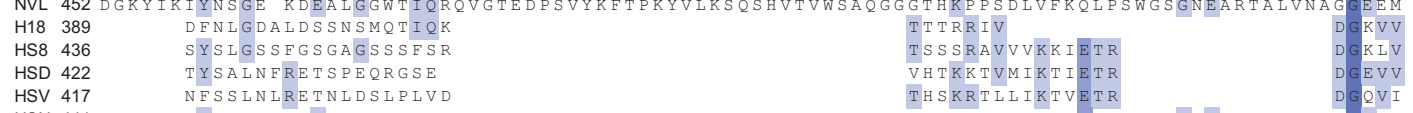

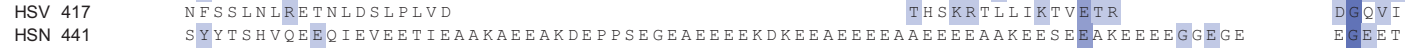

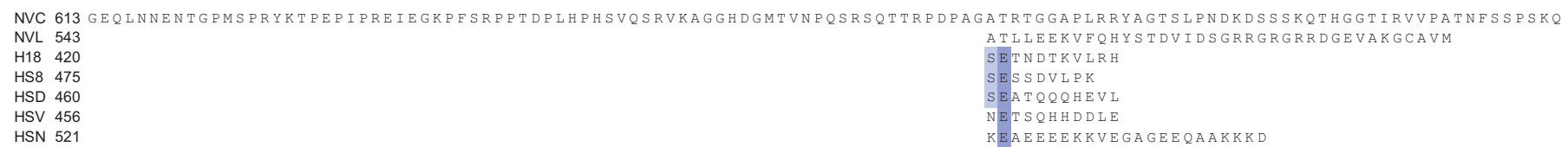

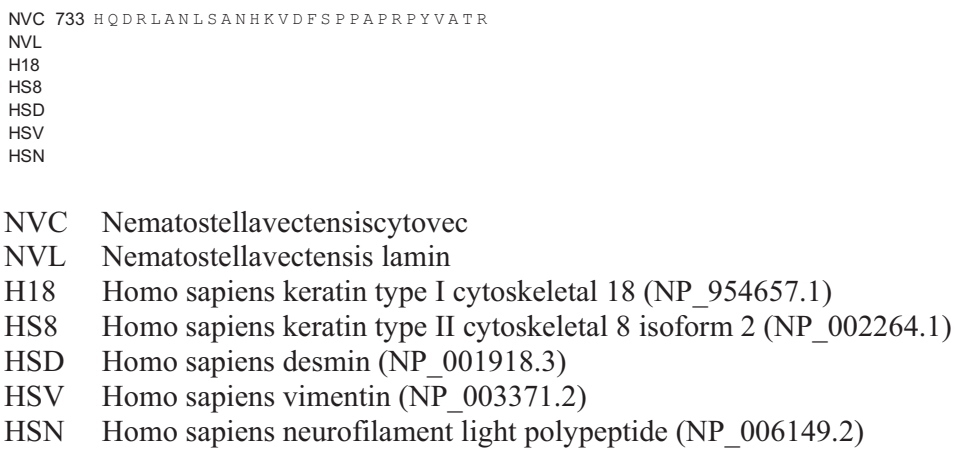

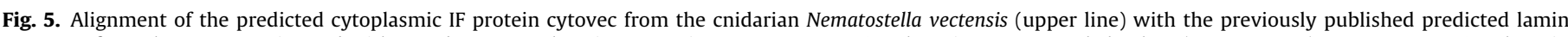

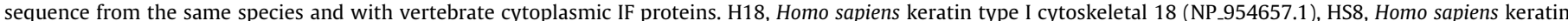

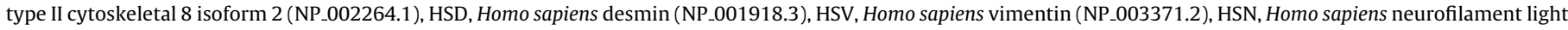
polypeptide (NP_006149.2). Note that sequence conservation is restricted to start of coil $1 \mathrm{~A}$ and the end of coil $2 \mathrm{~B}$. 


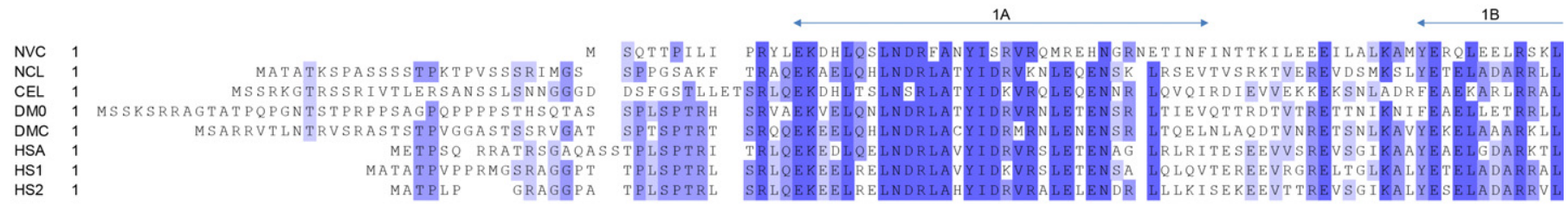

1B $\longleftarrow 2 \mathrm{~A}$

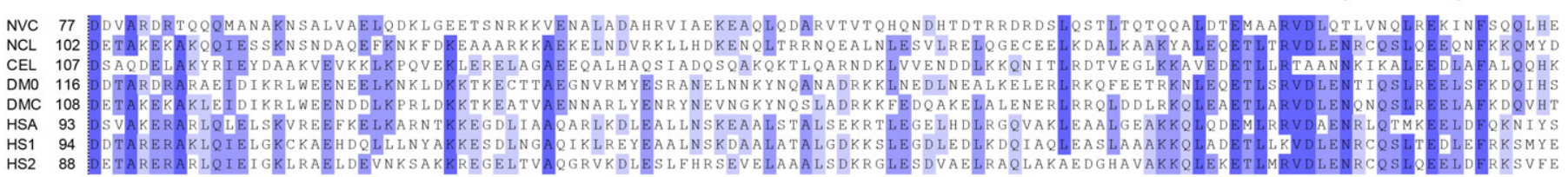

$2 \mathrm{~A}$

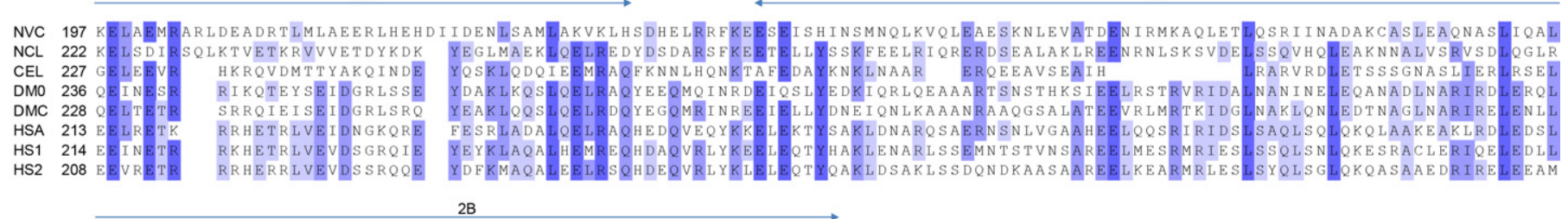

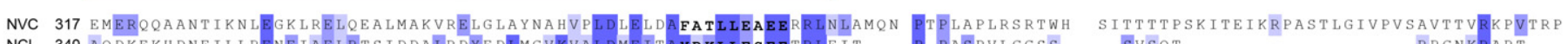
NCL 340 AQDKEKHDNEILLRENEIAELRTSIDDALRDYEDMGVKVALDMEITAYRKLLESEETRLEIT P PASPVLGSS SVSQT CEL 328 DTLKRSEQEKLDDKDARIAELNQEIERMMSEFHDLDVKIQLDAELKTYQALLEGEEERLNLTQEA PQNTSVHHSFS STGAS ARRGVKRRVV DMO 351 DNDRERHGQEIDLEKELIRLREEMTQQLKEYQDLMDIKVSLLEIAAYDKLLVGEARLNIT PATNTATVQSFS SLRNSTRATPSRR TPSAAVKRKRAVV

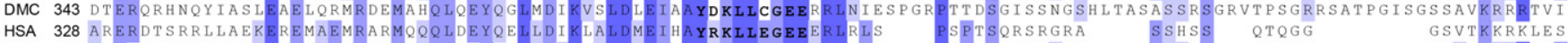

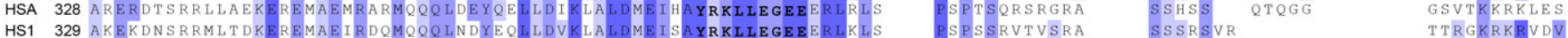

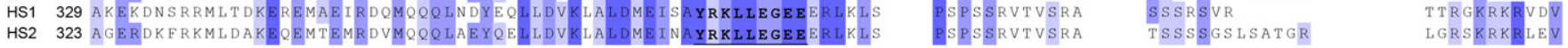

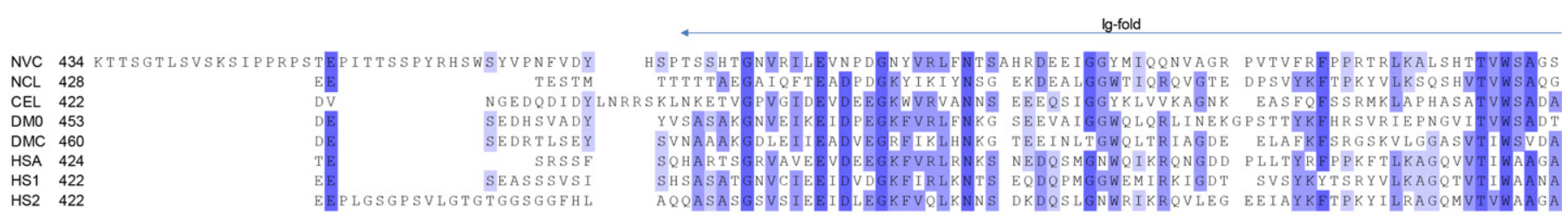
lg-fold

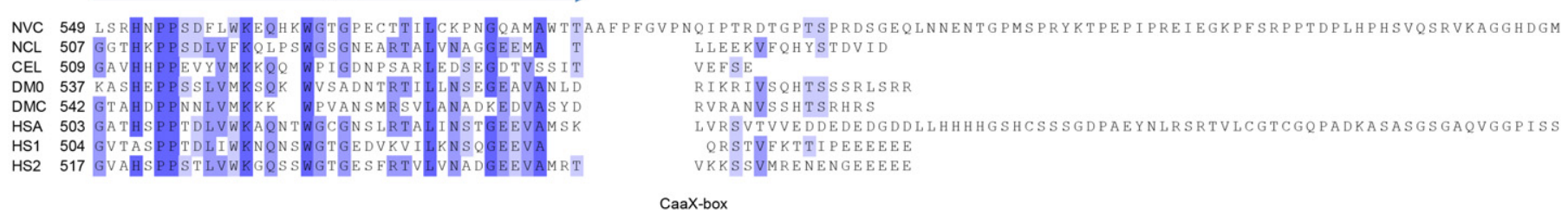

NVC 669 TVNPQSRSQTTRPDPAGARTGGAPLRRAGTSLPNDKDSSSKQTHGGTIRVVPATNFSPSKQHQDRLANLSANHKVDESPAPRPYVATR

NCL 561 SGRRG RGRRGEVAKGCAVM

CEL 553

DMO $594 \quad$ RSVTAVDGNEQLHQ Q GDPQQSN EKCAIM

HSA 614 GSSASSVTVTRSYRSVGGSGGSFGDLVTRSLLGNSPRTQSPQNCSIM

HS1 558 EEAAGVVEEELFH QGTPRASN RSCAIM

HS2 575 EAEFG EEDLFHQ QGDPRTS RGCYVM

NVC Nematostellavectensiscytovec

NCL Nematostellavectensis lamin

CEL Caenorhabditis elegans lamin (Q21443)

DM0 Drosophila melanogaster lamin Dm0 (P08928)

DMC Drosophila melanogaster lamin C (NP_523742.2)

HSA Homo sapiens lamin A/C transcript variant 1 (AAW32540.1)

HS1 Homo sapiens lamin B1 (AAC37575.1)

HS2 Homo sapiens lamin B2 (AAH06551.1)

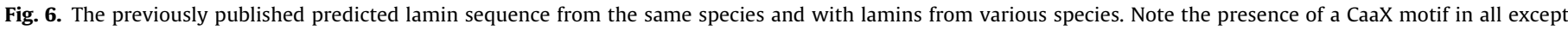

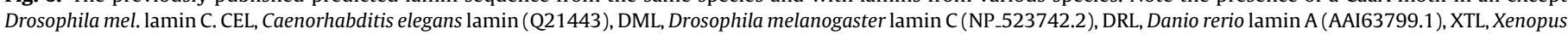
tropicalis lamin A/C (NP_001039148.1), HSL, Homo sapiens lamin A/C transcript variant 1 (AAW32540.1). 
using the intensive modeling Method. Visualized are the IG-folds by RCSB PDB Protein Workshop 3.9.

For the reconstructing of phylogenetic tree we used the maximum likelihood approach, which is available in SeaView v 4.3.0 by using the PhyML package v 3.0.1 (http://pbil.univ-lyon1.fr/ software/seaview.html). The calculated tree was stored in the svg format and afterword modified in Inkscape (http://inkscape. org) for a better visualization.

\section{Results}

\section{Identification of a cytoplasmic nematostella IF protein}

Using the previously described IF gene from the hemichordate S. kowalevskii (Zimek and Weber, 2002) as a probe on the genome information of Nematostella, we located a potential IF gene on scaffold 94 (nt. 50739-65390). The deduced protein sequence is shown in Fig. 1, which also gives the position of the 12 introns from the $5^{\prime}$-end. Intron sizes are $800 \mathrm{bp}, 285 \mathrm{bp}, 930 \mathrm{bp}, 380 \mathrm{bp}, 1554 \mathrm{bp}$, 110 bp, 5716 bp, 322 bp, 697 bp, 211 bp, 957 bp and 435 bp. Position of introns $1,2,3,4,5,8,9$ and 10 are conserved with those in Nematostella lamin (Zimek and Weber, 2008). Cytovec shares $19.7 \%$ identical amino acids with the previously described lamin gene of Nematostella, and $\sim 23.5 \%$ with the cytoplasmic nematocilins of $H$. vulgaris (Fig. 1). The highest degree of sequence identity is found at the rod end domains and in the middle of coil 1B. The IF consensus sequence YRKLLEGEE at the rod end is well conserved except that the Tyr residue is replaced by a Phe in cytovec. The Nematostella cytovec gene is clearly not a second lamin gene, since it lacks the nuclear localization signal and the C-a-a-M motive indicated for the lamin genes. However, it contains a recognizable immunoglobulin-like domain although structure prediction algorithms predict an arrangement distinctly different from that of Nematostella lamin (Fig. 2). Whereas the nuclear localization signal in bilateral metazoa involves 4 consecutive basic residues, the lamins from the two species of the radiata currently known show only 3 basic residues in the sequences KRSR (Hydra) and KRAR (Nematostella) (Zimek and Weber, 2011). The deduced protein sequence of 760 amino acids corresponds to a size of $85.4 \mathrm{kDa}$ and shows a length like in protostomic metazoa (Zimek and Weber, 2008). The sizeable number of Nematostella EST sequences covering the entire open reading frame support its expression. Table 1 lists the amino acid distribution in the two known Nematostella proteins. Across the rod domain, Nematostella lamin has a much higher ratio of acidic/basic (42.9\%) residues compared to cytovec (34.2\%). Another distinguishing feature is the prevalence of Ser/Thr residues in the lamin head domain. The overall amino acid distribution differs strongly from that of human keratins (Strnad et al., 2012).

\section{Relationship of nematostella cytovec to IF proteins from other species}

Fig. 3 reveals a distinct relationship between the 2 Nematostella IF proteins, the recently described isomin (I. maculatus; Mencarelli et al., 2011) and cytoplasmic IF proteins from C. elegans. The relationship among the Nematostella IF sequences $(\sim 19.6 \%)$ is closer than to C. elegans proteins ( $\sim 9.9-14 \%$ identity) with well conserved rod motifs at the start of coil $1 \mathrm{~A}$ and the end of coil $2 \mathrm{~B}$. Isomin is very distantly related to cytovec and shares $\sim 7.7 \%$ identical amino acids. To further examine the relationship between cytovec, cytoplasmic and nuclear IF proteins, additional comparisons were performed. Figs. 4-6 provide data on cytovec's relationship with selected IF sequences. The limited EST sequences from the anthozoan

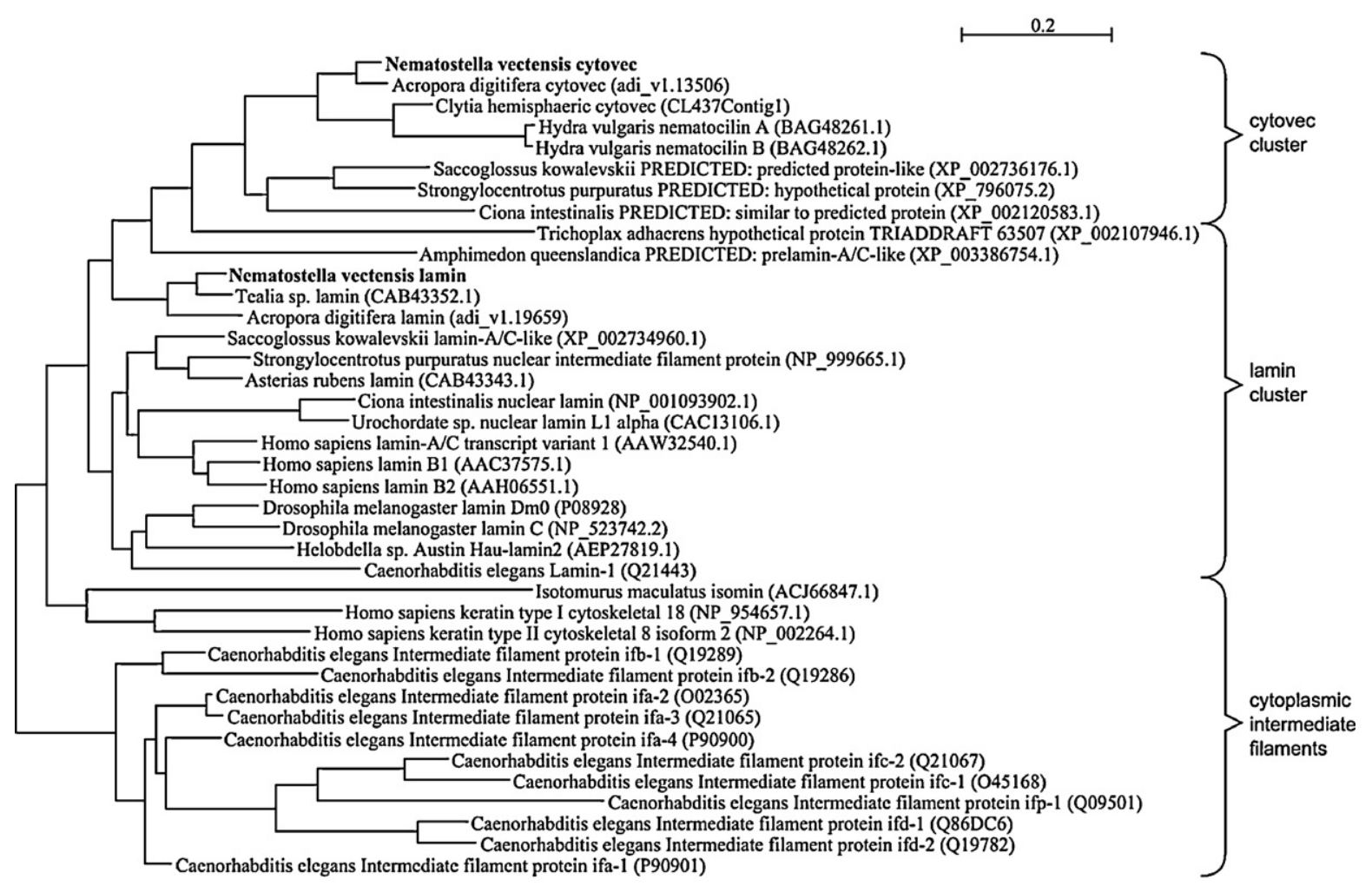

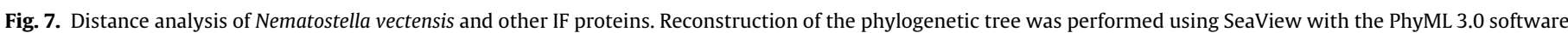

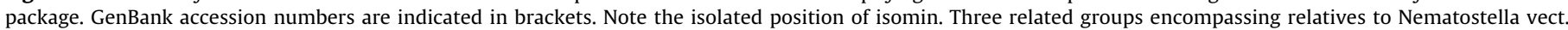

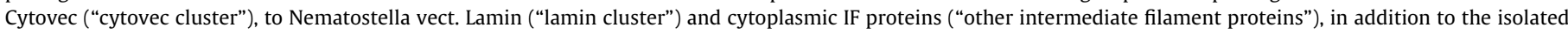
isomin, are recognized. 
A. digitifera and the hydrozoan Clytia hemispherica reveal $44.8 \%$ sequence identity between the entire Clytia protein and the first 627 amino acids of cytovec (Fig. 4). The C-terminal extension of 135 residues in the latter have no counterpart in the Acropora and Clytia sequences. With regard to other IF proteins, the extent of sequence identity is low (between $9 \%$ and $11.8 \%$ ). The rod end domains, in particular the end of coil $2 \mathrm{~B}$, are well conserved, supporting the classification of cytovec as cytoplasmic IF protein. Because of the closeness of these values it is currently impossible to decide which mammalian IF protein is the obvious counterpart of Nematostella IF cytovec. Attempts to get information via flanking genes as previously done for lamin genes (Zimek and Weber, 2011) were not possible because of the gaps in the genome of Nematostella. We note however that in the primitive bilateral animal, the nematode $C$. elegans, there is firm in vivo and in vitro evidence for a keratin-like obligatory heteropolymer system, but no indication for an additional homopolymer system (Karabinos et al., 2003). The phylogenetic tree analysis (Fig. 7) supports a relatively close relationship between cytovec, the cytoplasmic IF proteins of the anthozoan $A$. digitifera, the hydrozoans Clytia hemispherica, H. vulgaris, S. purpuratus, S. kowalevskii and C. intestinalis. It further confirms the grouping of Nematostella lamin with other lamins. These two groups are distantly related from other cytoplasmic IF proteins and from isomin.

\section{Discussion}

Among cytoskeletal proteins, intermediate filament proteins represent the most recent acquisition in evolutionary terms, coinciding with the occurrence of multicellularity (Wickstead and Gull, 2011). Using as probe a gene from metazoa closer related to radiata than the mammalian genes, we identified the putative cytoplasmic protein cytovec in Nematostella, which reveals that cytoplasmic intermediate filament genes are present in the common ancestor of Cnidaria and Bilateria. Sequence comparisons showed that this protein is related to the bona fide IF proteins nematocilin $A$ and $B$ of $H$. vulgaris and to the cytoplasmic IF proteins of the anthozoan $A$. digitifera and the hydrozoans $C$. hemispherica. The organization of the nematocilins in a central filament of the cnidocil of $H$. vulgaris where they are surrounded by microtubules suggest an architectural role of IF early in evolution (Hwang et al., 2008). Whether cytovec forms typical IF remains to be analyzed. Possibly, expression of a cytovec cDNA in E. coli may help to address its filament-forming capacity. The proteins could be purified by standard conditions and their assembly properties studied. We suggest that a further screen for cytoplasmic IF genes should be made when the genome of Nematostella is completed. The closest metazoan phyla for which abundant cytoplasmic intermediate filament proteins have been documented by electron microscopy are the Cnidarians and Ctenophora (Bartnik and Weber, 1989). It will be interesting to see whether evolution of cytoplasmic IF proteins coincides or follows metazoan evolution.

\section{Acknowledgements}

We thank Dr. Mary Osborn, this institute, for helpful comments on the manuscript. Cornelia Paz provided excellent secretarial help. This study was supported by the "Fonds der Chemischen
Industrie" to Klaus Weber. Work in the Magin lab is supported by the DFG (MA-1316), the BMBF (network EB) and the Translational Center for Regenerative Medicine, TRM, Leipzig, PtJ-Bio, 0315883.

\section{Appendix A. Supplementary data}

Supplementary data associated with this article can be found, in the online version, at http://dx.doi.org/10.1016/j.ejcb.2012.08.003.

\section{References}

Bartnik, E., Weber, K., 1989. Widespread occurrence of intermediate filaments in invertebrates; common principles and aspects of diversification. Eur. J. Cell Biol. 50, 17-33.

Bonifas, J.M., Rothman, A.L., Epstein, E.H.J., 1991. Epidermolysis bullosa simplex: evidence in two families for keratin gene abnormalities. Science 254, 1202-1205.

Dodemont, H., Riemer, D., Weber, K., 1990. Structure of an invertebrate gene encoding cytoplasmic intermediate filament (IF) proteins: implications for the origin and the diversification of IF proteins. EMBO J. 9, 4083-4094.

Doring, V., Stick, R., 1990. Gene structure of nuclear lamin LIII of Xenopus laevis; a model for the evolution of IF proteins from a lamin-like ancestor. EMBO J. 9, 4073-4081.

Fuchs, E., Weber, K., 1994. Intermediate filaments: structure, dynamics, function, and disease. Annu. Rev. Biochem. 63, 345-382.

Gruenbaum, Y., Margalit, A., Goldman, R.D., Shumaker, D.K., Wilson, K.L., 2005. The nuclear lamina comes of age. Nat. Rev. Mol. Cell Biol. 6, 21-31.

Hesse, M., Magin, T.M., Weber, K., 2001. Genes for intermediate filament proteins and the draft sequence of the human genome: novel keratin genes and a surprisingly high number of pseudogenes related to keratin genes 8 and 18. J. Cell Sci. 114 2569-2575.

Hwang, J.S., Takaku, Y., Chapman, J., Ikeo, K., David, C.N., Gojobori, T., 2008. Cilium evolution: identification of a novel protein, nematocilin, in the mechanosensory cilium of Hydra nematocytes. Mol. Biol. Evol. 25, 2009-2017.

Iwatsuki, H., Suda, M., 2010. Seven kinds of intermediate filament networks in the cytoplasm of polarized cells: structure and function. Acta Histochem. Cytochem. 43, 19-31.

Karabinos, A., Schmidt, H., Harborth, J., Schnabel, R., Weber, K., 2001. Essential roles for four cytoplasmic intermediate filament proteins in Caenorhabditis elegans development. Proc. Natl. Acad. Sci. U. S. A. 98, 7863-7868.

Karabinos, A., Schulze, E., Schunemann, J., Parry, D.A., Weber, K., 2003. In vivo and in vitro evidence that the four essential intermediate filament (IF) proteins A1, $\mathrm{A} 2, \mathrm{~A} 3$ and $\mathrm{B} 1$ of the nematode Caenorhabditis elegans form an obligate heteropolymeric IF system. J. Mol. Biol. 333, 307-319.

Mencarelli, C., Ciolfi, S., Caroti, D., Lupetti, P., Dallai, R., 2011. Isomin: a novel cytoplasmic intermediate filament protein from an arthropod species. BMC Biol. 28, 9-17.

Strnad, P., Usachov, V., Debes, C., Gräter, F., Parry, D.A., Omary, M.B., 2012. Unique amino acid signatures that are evolutionarily conserved distinguish simple-type, epidermal and hair keratins. J Cell Sci. 124, 4221-4232 (Epub 2012, Jan 3).

Szeverenyi, I., Cassidy, A.J., Chung, C.W., Lee, B.T., Common, J.E., Ogg, S.C., Chen, H., Sim, S.Y., Goh, W.L., Ng, K.W., Simpson, J.A., Chee, L.L., Eng, G.H., Li, B., Lunny, D.P., Chuon, D., Venkatesh, A., Khoo, K.H., McLean, W.H., Lim, Y.P., Lane, E.B., 2008. The human intermediate filament database: comprehensive information on a gene family involved in many human diseases. Hum. Mutat. 29, 351-360.

Vassar, R., Coulombe, P.A., Degenstein, L., Albers, K., Fuchs, E., 1991. Mutant keratin expression in transgenic mice causes marked abnormalities resembling a human genetic skin disease. Cell 64, 365-380.

Wehner, R., Gehring, W., 2007. Zoologie, 24th ed. Georg Thieme Verlag, Stuttgart, New York.

Wickstead, B., Gull, K., 2011. The evolution of the cytoskeleton. J. Cell Biol. 194, 513-525.

Windoffer, R., Beil, M., Magin, T.M., Leube, R.E., 2011. Cytoskeleton in motion: the dynamics of keratin intermediate filaments in epithelia. J. Cell Biol. 194, 669-678.

Zimek, A., Weber, K., 2002. The gene for a cytoplasmic intermediate filament (IF) protein of the hemichordate Saccoglossus kowalevskii; definition of the unique features of chordate IF proteins. Gene 288, 187-193.

Zimek, A., Weber, K., 2008. In contrast to the nematode and fruit fly all 9 intron positions of the sea anemone lamin gene are conserved in human lamin genes. Eur. J. Cell Biol. 87, 305-309.

Zimek, A., Weber, K., 2011. Flanking genes of an essential gene give information about the evolution of metazoa. Eur. J. Cell Biol. 90, 356-364. 